\title{
Amniotic Fluid Ingestion Enhances Opioid-Mediated But Not Nonopioid-Mediated Analgesia ${ }^{1}$
}

\author{
MARK B. KRISTAL, JOHN A. TARAPACKI AND DEBRA BARTON \\ Department of Psychology, State University of New York at Buffalo, Buffalo, NY 14260
}

Received 4 August 1989

\begin{abstract}
KRISTAL, M. B., J. A. TARAPACKI AND D. BARTON. Amniotic fluid ingestion enhances opioid-mediated but not nonopioid-mediated analgesia. PHYSIOL BEHAV 47(1) 79-81, 1990.-Ingestion of amniotic fluid or placenta by rats has been shown to enhance several types of opioid-mediated analgesia: that induced by morphine, footshock, vaginal/cervical stimulation, and late pregnancy. This enhancement has also been blocked by administration of opioid antagonists. The present study was designed to examine further the specificity of the enhancement effect for opioid-mediated analgesia by testing for enhancement following administration of aspirin, a nonopioid analgesic. The formalin test was used as the pain threshold assay. Amniotic fluid or beef bouillon was administered by orogastric tube to rats that were treated either with morphine sulfate or saline, or pretreated with naltrexone, then treated with aspirin or vehicle. Both morphine and aspirin treatments produced analgesia. Amniotic fluid significantly enhanced the analgesia produced by morphine, but did not enhance the analgesia produced by aspirin, further suggesting that the enhancing effect of amniotic fluid ingestion is specific for opioid-mediated analgesia, such as that existing at the start of parturition.
\end{abstract}

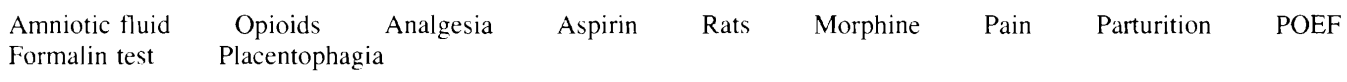

INGESTION of amniotic fluid or placenta by rats has been shown to enhance various types of existing analgesia, such as that produced by morphine injection $(12,13,15,16)$, footshock (16), vaginal/cervical stimulation (15), and late pregnancy (14). Ingestion of these substances in the absence of detectable analgesia, however, does not produce analgesia $(12,13,15,16)$. The evidence indicates conclusively that the active substance in amniotic fluid and placenta enhances opioid-mediated analgesia: all the forms of analgesia tested were completely or partially opioidmediated, and treatment with opioid antagonists such as naloxone and naltrexone not only blocked the analgesia, but also rendered the enhancer ineffective $(13,16)$. However, we do not know whether the enhancer is limited to opioid-mediated analgesia. The present experiment was undertaken, therefore, to determine whether ingestion of amniotic fluid enhances analgesia mediated by a nonopioid mechanism, that produced by aspirin $(8,9,19)$.

All the previous tests of the enhancer (which we now refer to as POEF, for Placental Opioid-Enhancing Factor) were conducted using the tail-flick latency (TFL) test. The literature suggests that with nonopioid analgesics. however, assays involving inflammation pain are more useful than the tail-flick latency test $(8-10,19)$. We therefore used a procedure that involves behavioral responses to the inflammation produced by subcutaneous injection of a small amount of formalin $(1-5,7,9,10,11,17,19)$.
An additional benefit of using the formalin test is that it provided an opportunity to test for enhancement of morphinemediated analgesia by POEF in a pain threshold assay other than the tail-flick latency test. The two assays differ in many respects $(3,11)$. The TFL test is a spinally mediated reflex, and its latency represents a simple, thermal pain threshold. On the other hand. the formalin test, which permits freedom of movement, elicits complex, integrated behavior in response to pain produced by a chemical stimulus associated with inflammation. The assays also differ in duration. The TFL test involves brief. rapidly rising pain. The time course of the pain produced by formalin injection. however, is biphasic: initially, pain subsides in $5-10 \mathrm{~min}$. but reappears $10-15 \mathrm{~min}$ later and persists for $1-2 \mathrm{hr}(2,7.9,10,11)$. In addition, the physiological mechanisms underlying the pain produced by brief exposure to a noxious stimulus, as in the TFL test, may be different from those involved in the tonic pain of the formalin test (17).

\section{METHOD}

\section{Subjects}

Ninety-six female Long-Evans rats, averaging 310.2 $\pm 4.2 \mathrm{~g}$. were used. They were housed in hanging, galvanized, wire-mesh cages $(24.5 \times 18 \times 18 \mathrm{~cm})$, in a colony maintained on a $14 \mathrm{hr} \mathrm{on} / 10$

'This research was supported by NSF grant BNS86-01818 awarded to M. B. K. 
hr off light cycle (lights on at $0500 \mathrm{hr}$, EST). All testing was conducted during the light phase. Except for the three hours prior to testing, and during testing, the rats received ad lib access to water and chow (Agway Prolab Rat/Mouse/Hamster Formula 3000 ). Because novel environments have been shown to reduce pain during the formalin test (1), all rats were intubated, without the administration of fluid, and habituated to the test chambers for $1 \mathrm{hr} /$ day, for at least the 5 days preceding testing.

\section{Apparatus}

Testing was conducted in clear glass aquaria $(51 \times 26 \times 32 \mathrm{~cm})$. A mirror was positioned at a $45^{\circ}$ angle below each glass-bottomed aquarium, and a tri-fold mirror was placed behind each aquarium, providing an unobstructed view of the rat's paws. Experimental and control fluids were administered through an orogastric tube consisting of $11.5 \mathrm{~cm}$ of PE 160 tubing fitted to a blunted 18-ga hypodermic needle, attached to a 1-cc glass syringe.

Data were recorded by pressing keys on the numeric pad of the keyboard of an IBM OS/2 Model 50 computer. Four keys corresponded to the behaviors of paw licking, paw lifting, freezing, and other, although ultimately the number of seconds spent licking and lifting was used as the dependent variable. The program that processed the data was designed for this experiment, and recorded durations over $30-\mathrm{sec}$ intervals. It is particularly useful for assays in which intensities of treatment are to be inferred from durations of behaviors. The details of the program are available elsewhere (18).

\section{Procedure}

The 96 rats were divided evenly among eight groups: four groups were used in the aspirin condition, and four in the morphine condition.

Aspirin condition (ASA). Each subject was deprived of food and water for $3 \mathrm{hr}$ prior to testing. To insure that opioid-mediated analgesia mechanisms were not involved, each rat received an injection of the opioid antagonist naltrexone $(1 \mathrm{mg} / \mathrm{kg}, \mathrm{SC})$ and was returned to its home cage. Although naltrexone does not block all opioid systems, it was chosen a) because of its long-lasting effect, and b) based on previous work in our laboratory, $1.0 \mathrm{mg} / \mathrm{kg}$ naltrexone is sufficient to block analgesia produced by $3 \mathrm{mg} / \mathrm{kg}$ morphine (11). Thirty minutes later, half the subjects received an injection of aspirin $(150 \mathrm{mg} / \mathrm{kg}$, IP, in a volume of $1 \mathrm{ml})$. The dose of aspirin was chosen after pilot tests showed that this dose produced a detectable but still enhanceable level of analgesia. The aspirin (acetylsalicylic acid, Sigma Chemical, St. Louis, MO) was dissolved in a sufficient amount of $2 \mathrm{~N} \mathrm{NaOH}$ to bring the $\mathrm{pH}$ to 6.95 , then was brought up to volume by the addition of $0.1 \mathrm{M}$ Tris buffer. The remaining rats in the aspirin condition received an IP injection of an equal volume of the $\mathrm{NaOH} /$ Tris vehicle, matched for $\mathrm{pH}$ by the addition of acetic acid.

Morphine condition (MS). All rats in the morphine condition first received an injection of physiological saline $(1 \mathrm{mg} / \mathrm{kg}$, IP), as a control for the naltrexone injection administered to all the rats in the ASA condition. Thirty minutes later, two of the four MS groups received an injection of a threshold dose of morphine sulfate $(3 \mathrm{mg} / \mathrm{kg}$. IP); the rats in the remaining two groups received an injection of physiological saline (Sal: $1 \mathrm{ml} / \mathrm{kg}$, IP) as a control. This dose of morphine was selected because it produced a small but reliable analgesia in about half the subjects $(11,13)$.

Formalin test. Thirty minutes after injection of naltrexone (ASA condition) or saline control (MS condition), each rat received the injection of analgesic or vehicle. Ten minutes later, each rat received an $0.05-\mathrm{ml}$ injection of $15 \%$ formalin under the dorsal skin of the right hindpaw, using a 26-ga hypodermic needle.

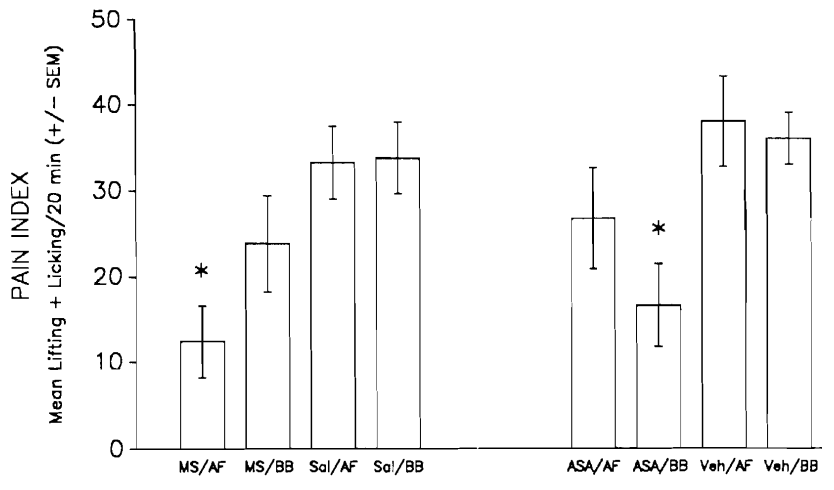

TREATMENT

FIG. 1. Pain index (mean sec \pm SEM spent paw licking and paw lifting over $20 \mathrm{~min}$ ) for rats in the morphine condition (morphine sulfate $=\mathrm{MS}$; saline control $=\mathrm{Sal}$ ) or the aspirin condition (aspirin $=\mathrm{ASA}$; vehicle control $=$ Veh) that were intubated with $0.25 \mathrm{ml}$ of either amniotic fluid (AF) or beef bouillon (BB). Rats in the aspirin condition were pretreated with naltrexone. $(n=12$ /group; *significantly different from Sal or Veh, $p<0.05$ ).

Fifteen minutes after that, each rat received an orogastric infusion of $0.25 \mathrm{ml}$ amniotic fluid (AF) or beef bouillon control (BB), and was placed in the testing chamber. Behavioral observations were begun 5 min after intubation ( $20 \mathrm{~min}$ after formalin injection), and were continued for $20 \mathrm{~min}$. The total number of seconds each rat spent lifting or licking its paw was accumulated as an index of pain and was subjected to statistical analysis.

To reduce the effect of the formalin injection, immediately after testing each rat was anesthetized with an injection $(0.65$ $\mathrm{ml} / \mathrm{kg}$, IP) of a $4: 1$ mixture of ketamine (Ketaset, $100 \mathrm{mg} / \mathrm{ml}$ ) and xylazine (Rompun, $20 \mathrm{mg} / \mathrm{kg}$ ).

Amniotic fluid was obtained surgically from 21-day-pregnant rats killed with $\mathrm{CO}_{2}(11,13)$. The fluid was quickly frozen until needed. For intubation, the fluid was warmed to $37^{\circ} \mathrm{C}$. Beef bouillon, the control fluid, was prepared from a commercial product (Wyler's Instant) in a strength half that recommended in the package directions. It was frozen and thawed after preparation. and otherwise treated identically to the $\mathrm{AF}(10,13)$.

\section{RESULTS}

Since the aspirin and morphine doses were not precisely equated for analgesic effect, the ASA and MS conditions were analyzed separately as $2 \times 2$ factorial ANOVAs [Drug (analgesic, vehicle $) \times$ Fluid $(\mathrm{AF}, \mathrm{BB}$ control)]. Additional probes were conduced using the Sheffé test.

The results of the aspirin and morphine treatments are presented in Fig. 1.

\section{Morphine Condition}

Morphine injection, overall, produced significantly more analgesia than did saline injection, $\mathrm{F}(1,44)=11.3, p<0.01$. AF ingestion, but not $\mathrm{BB}$ ingestion, significantly enhanced the analgesia produced by morphine; the MS/AF group showed significantly less pain than the saline controls ( $p<0.05$, Sheffé), whereas the MS/BB group did not $(p>0.05)$.

\section{Aspirin Condition}

Aspirin injection, overall, produced significantly more analge- 
sia than did vehicle injection, $\mathrm{F}(1,44)=9.96, p<0.01$, but this effect was attributable primarily to the effect of the ASA/BB treatment (ASA/BB vs. Veh/AF and Veh/BB, $p<0.05$, Sheffé). $A F$ ingestion did not increase the level of analgesia over that produced by $\mathrm{ASA} / \mathrm{BB}$, and in fact, produced a pain level between, and not significantly different from the ASA/BB group and the vehicle control groups $(p>0.05)$

\section{DISCUSSION}

Morphine injection, in the absence of amniotic fluid ingestion, did not produce a significant amount of analgesia, whereas aspirin injection, in the absence of amniotic fluid ingestion, did. This is not attributable to the greater efficacy of aspirin, but rather to the fact that the morphine and aspirin doses, as mentioned above, were not matched for analgesic potency. Since the morphine dose $(3 \mathrm{mg} / \mathrm{kg})$ was chosen because it was a threshold dose $(12,13)$, only about half the rats responded to it. However, whereas the magnitude of the analgesia exhibited by the rats responding to morphine was about the same as that exhibited by the rats responding to aspirin, the aspirin groups contained a much higher percentage of responders.

That amniotic fluid ingestion enhanced analgesia produced by morphine injection, as measured in the formalin test, is consistent with the enhancement effect found previously in the studies using the rat tail-flick assay $(12,13)$. Therefore, POEF activity is not specific to the neural and cutaneous mechanisms involved in the tail-flick latency procedure. That amniotic fluid ingestion did not enhance analgesia produced by aspirin is consistent with our contention $(12,13,15,16)$ that enhancement of analgesia produced by POEF (or, at least, by ingestion of amniotic fluid or placenta) is specific for opioid-mediated processes. It is possible. though, that the lack of enhancement of aspirin-mediated analgesia by $\mathrm{AF}$ ingestion might not generalize to other forms of nonopioidmediated analgesia.

These findings support the evidence suggesting that the relevance of placentophagia is that ingestion of birth fluids and afterbirth material (and therefore POEF) by parturient mammalian females enhances pregnancy-mediated analgesia immediately prior to, during, and immediately after delivery $(6,12-16)$.

\section{REFERENCES}

1. Abbott, F. V.: Franklin, K. B. J.; Connell, B. The stress of a novel environment reduces formalin pain: Possible role of serotonin. Eur. J. Pharmacol. 126:141-144; 1986.

2. Abbott, F. V.; Grimes, R. W.; Melzack, R. Single nerve capsaicin: Effects on pain and morphine analgesia in the formalin and foot-flick tests. Brain Res. 295:77-84; 1984

3. Abbott, F. V.; Melzack, R.; Samuel, C. Morphine analgesia in the tail-flick and formalin pain tests is mediated by different neural systems. Exp. Neurol. 75:644-651; 1982.

4. Calcagnetti, D. J.; Helmstetter, F. J.; Fanselow, M. S. Quaternary naltrexone reveals the central mediation of conditioned opioid analgesia. Pharmacol. Biochem. Behav. 27:529-531; 1987.

5. Dennis, S. G.; Melzack, R. Comparison of phasic and tonic pain in animals. In: Bonica, J. J.; Liebeskind, J. C.; Albe-Fessard, D. G.; Jones, L. E., eds. Advances in pain research and therapy. vol. 3. New York: Raven Press; 1979.

6. Doerr, J. C.; Kristal, M. B. Enhancement of opioid-mediated analgesia by ingestion of amniotic fluid: Onset latency and duration. Physiol. Behav. 46:913-915: 1989.

7. Dubuisson, D.; Dennis, S. G.. The formalin test: A quantitative study of the analgesic effects of morphine, meperidine, and brain stem stimulation in rats and cats. Pain 4:161-174; 1977.

8. Ferreira, S .H.; Lorenzetti, B. B.; Correa, F. M. A. Central and peripheral antialgesic action of aspirin-like drugs. Eur. J. Pharmacol. 53:39-48: 1978.

9. Hunskaar, S. Similar effects of acetylsalicylic acid and morphine on immediate responses to acute noxious stimulation. Pharmacol. Toxicol. 60:167-170; 1987

10. Hunskaar, S.; Hole, K. The formalin test in mice: Dissociation between inflammatory and noninflammatory pain. Pain 30:103--114; 1987.

11. Kocher, L. Systemic naloxone does not affect pain-related behavior in the formalin test in the rat. Physiol. Behav. 43:265-268; 1988.

12. Kristal, M. B.; Abbott, P.; Thompson, A. C. Dose-dependent enhancement of morphine-induced analgesia by ingestion of amniotic fluid and placenta. Pharmacol. Biochem. Behav. 31:351-356: 988.

13. Kristal, M. B.: Thompson, A. C.: Abbott, P. Ingestion of amniotic fluid enhances opiate analgesia in rats. Physiol. Behav. 38:809 815 1986.

14. Kristal, M. B.; Thompson, A. C.; Abbott, P.; DiPirro, J. M. Ferguson, E. J.; Doerr. J. C. Modification of periparturitional pain threshold by ingestion of amniotic fluid in the rat. Soc. Neurosci. Abstr. 14(Part 2):1158; 1988

15. Kristal, M. B.; Thompson, A. C.; Heller, S. B.; Komisaruk, B. R. Placenta ingestion enhances analgesia produced by vaginal/cervical stimulation in rats. Physiol. Behav. 36:1017-1020; 1986

16. Kristal, M. B.; Thompson, A. C.; Grishkat, H. L. Placenta ingestion enhances opiate analgesia in rats. Physiol. Behav, 35:481-486; 1985.

17. Ryan, S. M.; Watkins, L. R.; Mayer, D. J.; Maier, S. F. Spinal pain suppression mechanisms may differ for phasic and tonic pain. Brain Res. 334:172-175; 1985

18. Tarapacki, J. A.; Kristal, M. B. An adaptable microcomputer program for monitoring and recording behavior durations. Physiol. Behav., 47(2):in press; 1990.

19. Yaksh, T. L. Central and peripheral mechanisms for the antialgesic action of acetylsalicylic acid. In: Barnett, H. J. M.: Hirsh, J.; Mustard, J. F., eds. Acetylsalicylic acid: New uses for an old drug. New York: Raven Press; 1982 